\title{
EXPERIENCIAS
}

\section{PROYECTO BRUJULA-PROGRAMA TUTOR: ORIENTACIÓN EN LA FACULTAD DE CIENCIAS DE LA UCA}

\section{COMPASS PROJECT-TUTOR PROGRAM: GUIDANCE IN THE SCIENCE FACULTY AT THE UNIVERSITY OF CADIZ}

\author{
J. A. Álvarez Saura, M. García Basallote, V. García Moreno, \\ C. García Vázquez y I. de Ory \\ Universidad de Cádiz
}

\begin{abstract}
RESUMEN
En este artículo se intenta dar a conocer el proyecto Brújula, una iniciativa de tutorización en la Facultad de Ciencias de laUniversidad de Cádiz. Se analizan las motivaciones que hicieron posible su puesta en funcionamiento, cómo se ha desarrollado, y se analizan los resultados obtenidos en estos tres primeros años de vida.
\end{abstract}

Palabras clave: Tutoría y orientación universitaria, profesor tutor.

\section{ABSTRACT}

This paper presents the Brújula Project, a guidance program performed by the Faculty of Science, from University of Cadiz. The facts which motivate its beginning will be analysed, and besides the development and the results obtained through these last three years.

Key words: University Tutorial, Guidance system.

\section{Estructura del Proyecto-Programa y objetivos}

Para los alumnos de nuevo ingreso, la Universidad se revela los primeros meses como un medio hostil y extraño. Esta impresión se traduce en cierta desorientación, bien debida a deficiencias en la formación anterior, bien debida a las pautas de comportamiento que impone la nueva situación.

Durante el Bachillerato, la naturaleza de la relación que establece un alumno con sus profesores y orientadores suele ser bastante más personal de lo que, en la mayoría de los casos, se puede llegar a generar durante un primer curso de cualquier titulación universitaria. 
De esta relación, el alumno extrae la información necesaria para ajustar su curriculum a sus aspiraciones en cada momento.

Por otra parte, la actual universidad española se construye sobre unos planes de estudios que conceden a los alumnos gran capacidad de decisión para seleccionar qué materias deben cursar y cuándo quieren hacerlo, pero no introduce de forma institucional ningún elemento nuevo cuya misión sea el seguimiento de la trayectoria individual de cada alumno. En los últimos años, se ha observado un interés creciente de la comunidad educativa por la puesta en marcha de distintas medidas que redunden, de uno u otro modo, en una correcta integración del alumno en la vida universitaria, así como por optimizar la calidad de su formación. Dentro de este contexto, se ha implantado en la Facultad de Ciencias de la Universidad de Cádiz, un proyecto de mejora de la calidad, gracias a la participación voluntaria y coordinada de un grupo de sus profesores.

Este proyecto tuvo su origen en la evaluación de la Licenciatura de Química, realizada en 1996 en el marco del I Plan Nacional para la Evaluación de la Calidad de las Universidades. En su informe final se recogía una propuesta de mejora. Esta propuesta fue el origen del Proyecto Brújula, diseñado a finales del curso 97-98, cuyo nombre refleja claramente su vocación de orientar al alumno, insertando dentro del mismo el Programa Tutor, con el objetivo específico de cubrir la tutorización del alumnado. Para llevar a cabo este programa, se creó un grupo de trabajo (Grupo de Acción Tutorial) que elaboró ciertas estrategias que se vienen desarrollando desde entonces de manera ininterrumpida. Dado que la necesidad de orientación no era exclusiva de los estudiantes de Química, esta labor se amplió a las demás titulaciones con primer ciclo impartidas en la Facultad de Ciencias: Matemáticas e Ingeniero Químico.

\section{Composición del Grupo de Acción Tutorial}

Para desarrollar estas nuevas iniciativas, la primera medida fue solicitar personas con inquietudes que quisieran participar en este proyecto de forma desinteresada. Esta llamada tuvo una respuesta importante: comenzamos con un grupo de unos treinta voluntarios, aunque el número de personas implicadas ha ido aumentado ligeramente cada curso. Su composición actual es amplia, plural y, además, en todo momento se admite la incorporación de nuevos profesores. Como consecuencia de esta diversidad, se complementa la experiencia de los profesores más veteranos con las inquietudes de los más jóvenes.

La labor del Grupo es coordinada por una pequeña comisión constituida por un coordinador general del Programa, que gestiona la incardinación del Grupo dentro de la Facultad, así como coordinadores de cada una de las titulaciones participantes en el proyecto, que se encargan de la puesta en marcha del Programa en lo que se refiere a su titulación. Este grupo mantiene reuniones de forma periódica para hacer un seguimiento adecuado de las actividades.

El trabajo desarrollado se encuadra fundamentalmente en tres aspectos bien diferenciados. Por una parte, se encarga de construir actuaciones que consigan transmitir al alumno una información completa y eficaz en cada una de las etapas de su ciclo formativo. Por otra, complementa la relación directa que cada tutor establece con los alumnos, con el establecimiento de cauces eficaces para comunicarse con el resto de profesores participantes en el proyecto. Por último, cada curso se plantea la elaboración, realización y análisis de una encuesta, entre todos los alumnos del centro, para recoger su opinión sobre determinados pro- 
blemas que les afectan directamente. Las conclusiones de estos trabajos, así como las dificultades detectadas por el Programa Tutor, se presentan a las instancias universitarias adecuadas, junto con posibles actuaciones de mejora, y son punto de partida para la solución efectiva de estas cuestiones.

\section{Seminarios de información al alumnado}

Es evidente que existe una información de utilidad general para cualquier estudiante universitario y que debe, por tanto, comunicarse de manera que llegue a todos ellos en un plazo de tiempo adecuado. Sin embargo, los medios de difusión que habitualmente se utilizan (carteles, folletos, páginas en la red, etc) son a menudo ignorados por los alumnos, saturados, en muchos casos, ante la gran cantidad de contenidos que se les ofrecen. Ante tal vorágine, el alumno debería ser capaz de seleccionar aquéllos que le resulten más útiles en cada situación. Sin embargo, es bastante común que su actuación consista precisamente es postergar indefinidamente el momento de adquirir estos conocimientos de forma que la información acaba llegando al alumno tarde y distorsionada, de lo que se derivan una serie de problemas debidos exclusivamente a una estrategia de comunicación inadecuada.

Este problema adquiere tintes más graves a comienzos de la vida universitaria. Para subsanar estas deficiencias se seleccionan determinados temas, que se consideran importantes, y los miembros del Grupo de Acción Tutorial más adecuados para desarrollar estos contenidos en diversos seminarios. Estas presentaciones recogen con claridad cada uno de los aspectos que se desean transmitir. Han de captar rápidamente el interés del alumno, ser amenas y fáciles de seguir. De este modo, durante los tres años que lleva de funcionamiento el Programa, se han organizado en esta Facultad más de cincuenta seminarios referidos a contenidos muy diversos.

En el desarrollo de estas sesiones informativas aparecen dos bloques bien diferenciados. Uno de ellos se centra exclusivamente en dar la acogida a los alumnos al comienzo de cada curso. El otro recoge encuentros con un contenido más amplio, espaciados durante el año académico.

Probablemente, la realización de seminarios durante el período de matrícula es una de las actividades del Programa Tutor más agradecida, ya que provoca beneficios inmediatos sobre un conjunto de alumnos muy amplio: aclara sus numerosas dudas, alivia de forma inmediata la repetición de consultas en la secretaría del centro y ayuda a paliar errores en la cumplimentación de los impresos. Asimismo evitamos, en la medida de lo posible, la matriculación del alumno en asignaturas optativas y de libre elección totalmente inadecuadas para su nivel de conocimientos o para la configuración de un currículo ajustado a sus aspiraciones.

La información trasmitida se complementa con la organización de mesas de orientación durante todo el período de matrícula, gestionadas por alumnos de cursos superiores que han recibido una formación especial para esta tarea. Estas medidas son suficientes para que el proceso de matrícula de los alumnos se desarrolle de manera satisfactoria, tanto para ellos como para la propia Facultad.

En cuanto al resto de los encuentros suministran información sobre la vida cotidiana universitaria, así como aspectos específicos referidos a los planes de estudios de cada una de las titulaciones, atendiendo en la medida de lo posible las demandas de información concretas que se detectan a lo largo del curso. 
Aunque la utilidad de estos seminarios es evidente, también es claro que el principal problema con el que nos encontramos es alcanzar un punto de equilibrio entre el desinterés que los alumnos sienten si la serie de conferencias se prolonga y separa en el tiempo y la saturación de información. Así, si los seminarios se concentran en las primeras semanas del curso, disminuye su eficiencia respecto al objetivo propuesto. Por el contrario, si se distribuyen de forma espaciada a lo largo de muchos meses disminuyen drásticamente los altos niveles de asistencia que se consiguen en las primeras sesiones.

Para valorar la acogida que se les dispensa, cada sesión acaba con una pequeña evaluación de la actividad realizada, por lo que nos consta en todos los casos la valoración positiva de la iniciativa, así como la certificación de la utilidad de la información recibida.

\section{Desarrollo del Proyecto Tutor}

Las tutorías personalizadas tienen como objetivo lograr la atención del alumno para orientarles durante sus estudios de una manera personal, analizando las peculiaridades que plantea cada uno de los estudiantes. Debido al elevado número de alumnos matriculados en la Facultad, el programa prevé inicialmente atender tan sólo a los alumnos de nuevo ingreso, aunque poco a poco cada vez son más el número de alumnos de cursos posteriores que solicita la asignación de un tutor.

La asignación de este tutor se realiza a comienzo del curso, apenas concluye el plazo de matriculación y se plantea de forma que no sea considerada en ningún caso, como la imposición de un vigilante, de alguien a quien dar explicaciones y rendir cuentas del nivel de estudios sino, por el contrario, insistiendo en que el tutor es un punto de referencia, una ayuda para resolver sus dudas, para responder a todas sus preguntas, o para indicarle dónde debe acudir a encontrar respuesta. Asimismo, es interesante que en la confección de los grupos asignados a cada tutor se tenga en cuenta la heterogeneidad del propio colectivo de alumnos, de forma que para cada tutor surjan situaciones similares que enfrentar y enriquecer de ese modo la experiencia global del Grupo.

El objetivo fundamental del primer encuentro no es otro que la presentación del tutor a su grupo completo. Dado que el número de profesores que participan en el programa es elevado, es frecuente que el tutor no sea un profesor que les imparta docencia en el primer cuatrimestre. Este hecho que puede generar dificultades, se ha de convertir en una ventaja, ya que dota al tutor de una visión más objetiva ante los problemas que el alumno plantea. Además, éste se sentirá más relajado si no siente que está siendo juzgado por un profesor que posteriormente va a evaluarlo en sus conocimientos, consiguiéndose con ello un ambiente más cordial.

Otra de las metas que se plantean para ese encuentro inicial es conocer la motivación y las circunstancias que les han llevado a cursar esa titulación. También resulta interesante que planteen las primeras cuestiones que ya comienzan a inquietarles: el ritmo de las clases y de las horas de estudio, la falta de preparación que quizá puedan detectar, etc. Se trata de animarles a participar con ganas en la vida universitaria y a trabajar desde el primer día.

En lo que se refiere al resto de las entrevistas que tendrán lugar durante el año académico, no existe un formato claro. Se recomiendan al menos un par de encuentros más, bien en grupo o bien en forma de entrevistas personales, o combinando ambos sistemas, con el objeto de comentar las dificultades que les puedan ir surgiendo, solicitar estrategias de estudio 
y diseñar de este modo su currículum del modo más adecuado para reflejar sus preferencias o aspiraciones profesionales. El tutor puede ayudarle a reconocer y superar problemas, antes incluso de que el alumno descubra su importancia real.

La experiencia ha demostrado que la práctica totalidad de los alumnos acuden a su primera cita con el tutor y al menos la mitad fijan al menos una entrevista más a lo largo del curso.

\section{Tratamiento de la información dentro del Programa Tutor}

Conocer la situación real de los alumnos es fundamental para el desarrollo con calidad del programa. La política de información está coordinada por el Decanato de la Facultad de Ciencias de la Universidad de Cádiz y se aplica a todos los Programas del Proyecto Brújula. La información en el Programa Tutor se basa en encuestas realizadas a los alumnos, las cuales se han centrado hasta el momento en la selección de asignaturas optativas y de libre elección, así como en el análisis de las causas del absentismo en el aula. Como consecuencia directa de todos los datos obtenidos, han surgido distintas iniciativas. Para empezar, a partir del curso 98-99, entró en funcionamiento una página web, en la que se encuadra una Guía, donde se recoge una ficha actualizada de cada asignatura junto a su temario y normas de evaluación, horarios y fechas de exámenes, etc.

Otro hecho significativo (curso 1999-2000) detectado en estas encuestas fue la demanda de asignaturas que sirvieran para cubrir las deficiencias en la formación previa [1] de los alumnos. A raíz de estas peticiones, el año siguiente se ofrecieron dos nuevas asignaturas, de seis créditos cada una, que permitían a los alumnos de nuevo ingreso cursar contenidos de Matemáticas y Físicas con un nivel de $2^{\circ}$ de Bachillerato LOGSE. Estos créditos se ofertan como Libre Elección para los alumnos de la Facultad, de forma que pueden ir cubriendo este apartado de su matrícula.

Por último, durante el curso 2000-2001 se ha analizado la falta de asistencia de los alumnos a las aulas y las diferentes causas que provocan este absentismo [2]. De los datos obtenidos se ha derivado la puesta en marcha de un nuevo programa «Iniciativa PEP (Potenciación de las Enseñanzas Prácticas)» que pretende favorecer un método alternativo de evaluación del alumno, a partir de su trabajo en el aula a lo largo de todo el curso y que, poco a poco, parece que comienza a dar resultados.

Además, continuando con la vocación orientadora del Proyecto Brújula, se puso en marcha en mayo de 2000 una Oficina de Atención al Alumno, en la que los interlocutores son también alumnos de últimos cursos o recién titulados con objeto de facilitar la comunicación con sus compañeros. El funcionamiento de esta Oficina requiere de dos becarios a media jornada, que reciben su beca a través de un convenio del Vicerrectorado de Estudiantes y el Instituto Andaluz de la Juventud, permitiéndonos así un horario de apertura de mañana y tarde, incluyendo períodos de exámenes y matrículas.

En esta Oficina se recopila toda la información que el alumno pueda solicitar: becas, intercambios con otras universidades, convalidaciones de estudios, etc. En el caso en que, para el problema planteado, se desconozca la vía adecuada de actuación, la Oficina recaba el apoyo de los profesores del Grupo de Acción Tutorial a los que resulta más sencillo articular otros mecanismos de información para obtener la respuesta adecuada. 


\section{Difusión de las iniciativas de Acción Tutorial}

La experiencia ha tenido una acogida muy favorable, tanto entre los alumnos como entre los profesores de la Facultad, aunque la falta de referencias en este tipo de iniciativas ha motivado que el Programa haya sufrido diversas modificaciones intentando optimizar sus resultados. Los resultados obtenidos y el esfuerzo realizado por la Facultad en el desarrollo del Proyecto Brújula y de este Programa en concreto, motivaron la presentación del mismo en el año 2000 a la convocatoria «Acciones de Mejora» del Plan Nacional de la Calidad de las Universidades, donde obtuvo el segundo premio. Al mismo tiempo, se ha recibido un número elevado de consultas interesándose por los detalles de este programa, tanto desde otros centros de la Universidad de Cádiz, como desde otras Universidades españolas, realizándose presentaciones del proyecto a nivel nacional en distintos foros.

Es de esperar que la generalización de este tipo de actuaciones en distintas titulaciones y universidades, cada una de ellas con su particular problemática, conduzca a una rápida mejora de la calidad de este tipo de proyectos, así como al aprovechamiento máximo del esfuerzo invertido para conseguir los objetivos propuestos.

\section{Referencias bibliográficas}

[1] Álvarez Saura, J. A., Duque Casas, E., García Basallote, M, García Moreno, V, García Vázquez, C., de Ory Arriaga, I. y Rodríguez Blázquez, I. (2002). Asignaturas de nivelación: Una alternativa dentro de la libre elección, aceptado en la revista Innovación Educativa: Universidad de Santiago de Compostela.

[2] Álvarez Saura, J. A., Duque Casas, E., García Basallote, M, García Moreno, V, García Vázquez, C., de Ory Arriaga, I. y Rodríguez Blázquez, I. (2002). Causas de la falta de asistencia a clase en una facultad de ciencias: análisis de los resultados de encuestas personales. Enviado a la revista Bordon. 